\title{
VIGILÂNCIA DE INFECÇÃO DE SÍTIO CIRÚRGICO NO PÓS-ALTA HOSPITALAR DE CIRURGIA CARDÍACA RECONSTRUTORA
}

\author{
Vanessa Damiana Menis Sasaki ${ }^{1}$, Adilson Edson Romanzini' ${ }^{2}$, Ana Paula Marcielo de Jesus ${ }^{3}$, Edevã de \\ Carvalho ${ }^{4}$, João Junior Gomes ${ }^{5}$, Valquiria Barco Damiano ${ }^{6}$
}

\footnotetext{
${ }^{1}$ Pós-graduanda em Enfermagem. Enfermeira assistencial do ambulatório de especialidades de São José do Rio Preto. São Paulo, Brasil. E-mail: vanessasasaki@hotmail.com

${ }^{2}$ Especialista em Enfermagem na Unidade de Terapia Intensiva. São Paulo, Brasil. E-mail: adilsonfqm@hotmail.com

${ }^{3}$ Especialista em Enfermagem em Urgência e Emergência. São Paulo, Brasil. E-mail: anapaulamarcielo@bol.com.br

${ }^{4}$ Pós-graduando em Enfermagem. São Paulo, Brasil. E-mail: edi.enfermagem@hotmail.com

${ }^{5}$ Mestre em Promoção da Saúde. Professor Adjunto I da Universidade Paulista (UNIP). São Paulo, Brasil. E-mail: jjenf@terra. com.br

${ }^{6}$ Doutora em Microbiologia Aplicada. Professora Titular da UNIP. São Paulo, Brasil. E-mail: damianovb@unip.br
}

RESUMO: A incidência de doenças cardiovasculares vem aumentando a cada ano e, na maioria das vezes, a cirurgia de revascularização do miocárdio se faz necessária, sendo a infecção de sítio cirúrgico, uma das principais complicações. Este estudo teve por objetivo identificar sinais e sintomas de infecção de sítio cirúrgico no pós-alta hospitalar de cirurgia cardíaca reconstrutora. Trata-se de um estudo quantitativo descritivo de caráter prospectivo. A coleta de dados foi realizada na residência de 20 pacientes maiores de 18 anos, submetidos à cirurgia cardíaca reconstrutora, em um hospital filantrópico de nível quaternário, situado em São José do Rio Preto-SP Brasil. Quatro pacientes apresentavam exsudato seroso ou purulento e hiperemia na incisão cirúrgica. Evidenciou-se uma taxa de $20 \%$ de infecção de sítio cirúrgico no pós-alta hospitalar. Ressalta-se a necessidade de implementar um método de vigilância no período cirúrgico focalizado no pós-alta hospitalar que possibilite uma real notificação das infecções de sítio cirúrgico, já que estas podem ser subnotificadas ocasionando uma alta taxa de morbi-mortalidade.

DESCRITORES: Enfermagem. Sinais e sintomas. Infecção da ferida operatória. Cirurgia cardíaca.

\section{SURGICAL SITE INFECTION SURVEILLANCE IN POST-HOSPITAL DISCHARGE AFTER CARDIAC RECONSTRUCTIVE SURGERY}

\begin{abstract}
The incidence of cardiovascular disease has been increasing annually in recent years, and in the majority of cases Myocardial Revascularization surgery becomes necessary. One of the principle complications of this procedure is post-surgical infection of the surgical site. This study aimed to identify signs and symptoms of infection of surgical site in the post-hospital discharge following cardiac reconstructive surgery. This is a descriptive quantitative study of a prospective character. Data was collected in the respective houses of 20 patients older than 18 years old who were submitted to cardiac reconstructive surgery in a quaternary philanthropic hospital located in São José do Rio Preto, SP, Brazil. Four patients presented serous exudate or festering and hyperemia in the surgical incision. An infection rate of the surgical site of $20 \%$ was found in post-hospital discharges studied. We highlight the need to implement a surveillance method in the period following post-hospital discharge to enable a real notification of surgical site infections, as these may be underreported and cause a high mortality rate.
\end{abstract}

DESCRIPTORS: Nursing. Signs and symptoms. Surgical wound infection. Cardiac surgery.

\section{VIGILANCIA DE LA INFECCIÓN DE SITIO QUIRÚRGICO DESPUÉS DEL ALTA HOSPITALARIA DE CIRUGÍA CARDÍACA RECONSTRUCTIVA}

\begin{abstract}
RESUMEN: Cada año la incidencia de las enfermedades cardiovasculares ha ido en aumento, y en la mayoría de los casos, la cirugía de revascularización miocárdica se hace necesaria, siendo la infección de sitio quirúrgico una de las principales complicaciones. Este estudio tuvo como objetivo identificar señales y síntomas de infección de sitio quirúrgico después del alta hospitalaria de cirugía cardíaca reconstructiva. Este es un estudio cuantitativo descriptivo de carácter prospectivo. La recolección de datos se realizó en la residencia de 20 pacientes mayores de 18 años, sometidos a cirugía cardíaca reconstructiva en un hospital filantrópico cuaternario, de São José de Rio Preto-SP, Brazil. Cuatro pacientes presentaban exudado seroso o purulento e hiperemia en la incisión quirúrgica. Se encontró una tasa de $20 \%$ de infección de sitio quirúrgico después del alta hospitalaria. Destacamos la necesidad de aplicar un método de vigilancia en el período posterior al alta hospitalaria para permitir una información real de las infecciones del sitio quirúrgico, ya que estas pueden no ser reportadas, causando una alta tasa de morbilidad y mortalidad.
\end{abstract}

DESCRIPTORES: Enfermería. Signos y síntomas. Infección de herida operatoria. Cirugía cardíaca. 


\section{INTRODUÇÃO}

A incidência de doenças cardiovasculares nos países desenvolvidos vem aumentando a cada ano, com 80\% relacionadas à doença arterial coronariana, na qual, a maioria das vezes, a cirurgia de Revascularização do Miocárdio (RM) se faz necessária. No Brasil, as doenças cardiovasculares são responsáveis por cerca de $30 \%$ de todos os óbitos, sendo o infarto agudo do miocárdio sua principal causa. ${ }^{1}$

Recomenda-se cirurgia cardíaca quando a probabilidade de sobrevida com vida útil é maior com tratamento cirúrgico do que com tratamento clínico. Operações cardiovasculares estão entre os mais comuns procedimentos cirúrgicos realizados em grandes hospitais. Dentre as cirurgias cardíacas, a cirurgia reconstrutora é mais complexa e consome mais tempo do que as reparações simples, estas nem sempre são curativas e a reintervenção pode ser necessária. ${ }^{2}$

As principais complicações de cirurgia cardíaca são as infecções hospitalares, definidas como aquelas adquiridas após a internação do paciente e que se manifestam durante o período de hospitalização, ou mesmo após a alta quando puder ser relacionada com a internação ou procedimentos hospitalares. ${ }^{3}$ Dentre elas, estão as de sítio cirúrgico, seguidas por pneumonias, sepse, infecções relacionadas a cateteres e infecções do trato urinário. ${ }^{4}$

As Infecções de Sítio Cirúrgico (ISCs) ocorrem geralmente entre o $7^{\circ}$ ao $14^{\circ}$ dia de pós-alta hospitalar, sendo os agentes mais freqüentes os Staphylococcus sp e bacilos gram negativos. ${ }^{4}$ Uma infecção acresce, em média, cinco a 10 dias ao período de internação. Além disso, os gastos relacionados a procedimentos diagnósticos e terapêuticos da infecção fazem com que o custo do procedimento seja elevado. ${ }^{6}$

O alto custo das hospitalizações tem abreviado o tempo de internação e o planejamento da alta do paciente tem sido uma das principais preocupações para assegurar a continuidade do tratamento e evitar a reinternação. ${ }^{7} \mathrm{~A}$ alta precoce pode ser um problema para a detecção das infecções que se manifestam nos primeiros dias de pós-operatório, especialmente para aqueles procedimentos cujo tempo de permanência no hospital após a cirurgia é muito curto. ${ }^{8}$ A vigilância do paciente cirúrgico, na maioria das instituições, tem ocorrido apenas durante o período de internação. Estudos que restringem o seguimento do paciente cirúrgico durante o período de internação tendem a apre- sentar menores taxas de ISCs, quando comparados àqueles que incluem o seguimento depois da alta, o que implica num adicional esforço para o sistema de vigilância hospitalar. ${ }^{9}$

Desde a internação, o momento da alta hospitalar é o mais esperado pelo paciente e sua família. ${ }^{10} \mathrm{O}$ retorno para casa após a alta hospitalar é um momento de ansiedade para o paciente e familiares, uma vez que se sentem desprotegidos da vigilância constante da equipe de saúde fora do hospital. Assim, a alta hospitalar pode ser vista como uma ameaça para suas vidas, pois muitas dúvidas poderão surgir neste momento, tais como questões relacionadas ao tratamento, aparecimento e/ou manutenção de sinais e sintomas, e surgimento de novos problemas. ${ }^{8}$

A identificação precoce de complicações relacionadas ao período pós-operatório poderá auxiliar os enfermeiros na elaboração de intervenções adequadas às necessidades individuais de cada paciente, colaborando para a implementação de ações rápidas e eficazes para a resolução dos problemas identificados. Sendo assim, este estudo teve por objetivo identificar sinais e sintomas de infecção de sítio cirúrgico no pós-alta hospitalar de cirurgia cardíaca reconstrutora.

\section{METODOLOGIA}

Trata-se de um estudo quantitativo, descritivo, de caráter prospectivo. A população do estudo foi composta por todos pacientes submetidos à cirurgia cardíaca reconstrutora no período de junho a setembro de 2008, de ambos os sexos e idade maior ou igual a 18 anos. A pesquisa ocorreu em um hospital filantrópico de nível quaternário, situado em São José do Rio Preto-SP, que possui em média cerca de 3,5 mil internações/mês e 2,1 mil cirurgias/mês, sendo que destas, 15 são cirurgias cardíacas reconstrutoras.

Durante o período de internação, quatro pesquisadores, previamente treinados, realizaram acompanhamento diário de 37 pacientes que se submeteram a cirurgia cardíaca reconstrutora. A primeira etapa da coleta de dados teve como fonte os prontuários dos pacientes, registros médicos e de enfermagem. Foram coletados dados pessoais tais como: sexo e idade, endereço residencial, número de telefone, e sobre a ocorrência da ISC, que foram registrados em instrumento específico durante a internação. Foram excluídos da pesquisa aqueles pacientes que desenvolveram ISC intrahospitalar e os que não foram encontrados, devido aos dados incorretos de endereço e número de tele- 
fone, originando assim, a amostra de 20 pacientes. Conforme os pacientes recebiam alta hospitalar, foram convidados a participar da pesquisa por telefone, onde os mesmos agendaram os horários das visitas de acordo com sua disponibilidade. A vigilância pós-alta foi realizada por meio de visitas domiciliares, que ocorreram em diversas cidades do Estado de São Paulo entre o $7^{\circ}$ e o $14^{\circ}$ dia de pós-alta hospitalar, devido à maior incidência de infecção de sítio cirúrgico nesse período. ${ }^{11}$

A segunda etapa da pesquisa foi realizada após assinado o Termo de Consentimento Livre e Esclarecido. O instrumento elaborado para o levantamento de dados no período pós-operatório, sob a forma de questionário do tipo check-list é embasado nas orientações do Center of Deasese Controle $^{12}$ e Sociedade Brasileira de Infectologia ${ }^{6}$, em relação aos cuidados pós-operatórios com a incisão cirúrgica e o método de vigilância epidemiológica para a identificação das ISCs, classificadas como infecção superficial, profunda e de órgão/ espaço. Este instrumento avaliou a ferida cirúrgica como principal fonte de informação, considerando itens como: presença de dor ou hipersensibilidade local, edema localizado, eritema, calor local, odor, deiscência, fibrina, e exsudato purulento pela cicatriz. Também se considerou como infecção a manutenção ou introdução de outro esquema de antibioticoterapia por ocasião dos retornos ao ambulatório e diagnóstico de infecção pelo médico que acompanha o paciente. Caso no momento das visitas domiciliares, houvesse necessidade, foi realizado o curativo no paciente de acordo com a prescrição médica ou de enfermagem. Caso fosse constatada alguma alteração com o paciente, este seria orientado a procurar o serviço de saúde mais próximo da sua residência.

A análise dos dados foi feita na forma descritiva e utilizou-se de operações estatísticas de distribuição de freqüência e porcentagem. Os resultados evidenciados foram comparados com a literatura específica. A pesquisa foi aprovada pelo Comitê de Ética em Pesquisa da Faculdade de Medicina de São José do Rio Preto, sob o parecer $n^{\circ} 113 / 2008$.

\section{RESULTADOS E DISCUSSÃO}

Das cirurgias cardíacas realizadas, 90\% foram de revascularização do miocárdio e 10\% plastia de valvas, totalizando 37 procedimentos, sendo que 17 pacientes foram excluídos da pesqui- sa, pois 13 adquiriram infecção no intra-hospitalar e quatro não foram encontrados. Foram realizadas 20 visitas domiciliares em treze cidades da região de São José do Rio Preto. As visitas ocorreram entre o período do $7^{\circ}$ ao $14^{\circ}$ dia de pós-alta hospitalar, sendo que $55 \%$ dos pacientes encontravam-se entre o $10^{\circ}$ e $14^{\circ}$ dia de pós-alta.

Entre os pacientes visitados predominou o sexo masculino (70\%), a idade entre 43 a 53 anos $(45 \%)$ e o ensino fundamental incompleto $(50 \%)$. Destes, $70 \%$ eram casados e $50 \%$ trabalhadores braçais. Todos residiam em casas de alvenaria, possuíam saneamento básico e estas apresentavam boas condições de higiene.

Quanto aos aspectos da incisão cirúrgica, 85\% dos pacientes apresentavam incisão limpa e seca. A presença de deiscência, exsudato e hiperemia foram verificadas em $20 \%$, fibrina em $15 \%$, edema em $10 \%$, e calor, dor e odor em $5 \%$ dos pacientes (Gráfico 1). Dos pacientes que apresentaram exsudato, $50 \%$ era seroso e $50 \%$ purulento. Estes sinais e sintomas caracterizam infecção superficial.

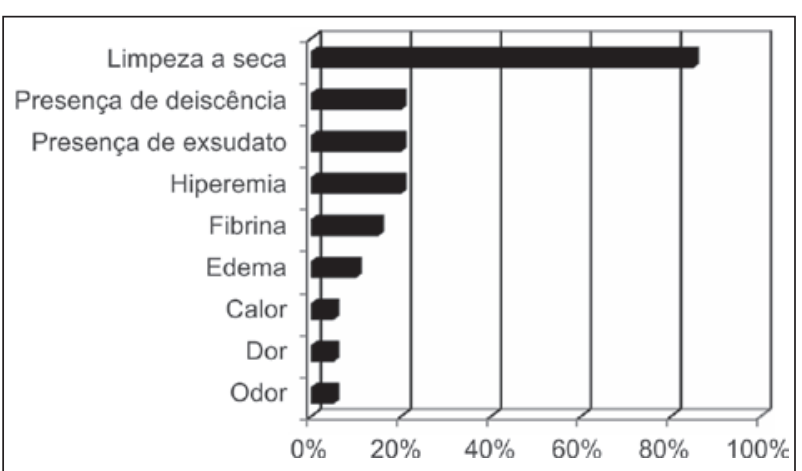

Figura 1 - Avaliação da incisão cirúrgica de cada paciente. São José do Rio Preto-SP, 2008

Infecção superficial é definida como sendo aquela que ocorre até 30 dias após a realização da cirurgia, envolve somente pele ou tecido subcutâneo e, no mínimo, um dos seguintes aspectos: drenagem purulenta com ou sem confirmação laboratorial; organismos isolados de cultura colhida de forma asséptica da secreção ou tecido superficial; sinais flogísticos ou incisão superficial aberta pelo cirurgião. ${ }^{13}$

Com a realização das visitas domiciliares do $7^{\circ}$ ao $14^{\circ}$ dia após a alta, foram evidenciados $20 \%$ de ISC. Desses, $15 \%$ eram do sexo masculino e tinham idade superior a 50 anos. Todos tinham doenças pré-existentes (tabela 1). 
Tabela 1 - Perfil dos pacientes com infecção de sítio cirúrgico segundo variáveis: sexo, fatores de risco, doenças pré-existentes e escolaridade. São José do Rio Preto-SP, 2008

\begin{tabular}{lc}
\hline Variáveis & $\begin{array}{c}\text { Proporção de } \\
\text { incidência (\%) }\end{array}$ \\
\hline Sexo & 75 \\
M & 25 \\
$\mathrm{~F}$ & \\
Idade & 25 \\
$\leq 50$ & 75 \\
$>50$ & \\
Escolaridade & 50 \\
Fundamental incompleto & 25 \\
Médio completo & 25 \\
Superior completo & \\
Índice de massa corpórea & 50 \\
$\leq 30$ & 50 \\
$>30$ & \\
Tabagismo & 25 \\
Sim & 75 \\
Não & \\
Doenças pré-existentes* & 100 \\
Sim & - \\
Não & \\
\hline
\end{tabular}

* Hipertensão arterial, Diabetes Mellitus ou Dislipidemia.

Diversos fatores predispõem o desenvolvimento da ISC que podem estar relacionados ao paciente, como idade, tabagismo, presença de doenças como obesidade, diabetes mellitus e outros. As alterações metabólicas causadas pelo diabetes mellitus são responsáveis pelo desenvolvimento de complicações importantes, como a deficiência na cicatrização que acomete os pacientes diabéticos com pobre controle glicêmico. Assim, tais fatores, podem ser entendidos como "características que estão associadas a um maior risco de adoecer", porém, a sua presença ou ausência não garante que a infecção ocorra. ${ }^{14-15}$

Do total de infecções evidenciadas no presente estudo, verificou-se uma prevalência de $76 \%$ de ISC até o $7^{\circ}$ dia de pós-alta e $23 \%$ do $8^{\circ}$ ao $14^{\circ}$ dia de pós-alta. De acordo com estudo publicado sobre vigilância em infecção de sítio cirúrgico, $82 \%$ de ISC foram evidenciadas até o $7^{\circ}$ dia de pós-alta e $11 \%$ do $8^{\circ}$ ao $14^{\circ}$ dia de pós-alta, sendo que $100 \%$ das infecções ocorreram durante o período de 30 dias de pós-alta hospitalar. ${ }^{11}$

A taxa de ISC em cirurgia cardíaca pode variar de 0,9 a $20 \%$ e está associada a altos índices de morbi-mortalidade..$^{12}$ Em uma pesquisa realizada sobre a vigilância pós-alta, verificou-se taxa global de $18 \%$ de ISC. Ainda observou-se um importante aumento desta taxa em quatro vezes quando a vigilância pós-alta foi realizada. Caso essa vigilância não fosse realizada, a taxa global de ISC seria de apenas de $4,5 \% .^{10}$

De acordo com a literatura, quando não existem ambulatórios de egressos, o melhor método para ser realizada a vigilância pós-alta, é pelo contato por carta ou telefone. Porém, por existir a dificuldade do profissional em realizar o diagnóstico de ISC por estes métodos, é possível suspeitar da confiabilidade da vigilância pós-alta para um identificador fidedigno, cuja incidência de ISC poderá ser sub ou hipernotificada. ${ }^{13}$

A vigilância pós-alta hospitalar por meio de visitas domiciliares destina-se a proporcionar ações mais integrais ao indivíduo e á família, e ainda uma integração entre os diversos níveis de saúde. É um método eficaz e meio de execução de técnicas ágeis e fidedignas de pesquisa ainda pouco divulgadas. ${ }^{16}$

Alguns autores diagnosticam ISC utilizando como padrão-ouro a presença de secreção purulenta, enquanto outros relatam que não há padrãoouro para o diagnóstico de ISC, pois o julgamento é subjetivo e sujeito á variações, dependendo muito das definições utilizadas, do conhecimento e do olhar individual de cada avaliador. . $^{8,13}$

O êxito para a vigilância e diagnóstico das ISC está diretamente relacionado com o envolvimento dos diversos níveis de saúde e de todos os profissionais que atuam na assistência ao paciente cirúrgico. A assimilação e implementação dos procedimentos corretos por quem assiste ao paciente e a necessária integração com a equipe da Comissão de Controle de Infecção Hospitalar é fundamental na prevenção e controle das infecções. Porém, de nada adianta o conhecimento do fenômeno e das medidas preventivas, se quem presta assistência não as adota no seu fazer profissional. ${ }^{3}$

\section{CONCLUSÃO}

Os sinais e sintomas de infecção de sítio cirúrgico no pós-alta hospitalar de cirurgia cardíaca reconstrutora, evidenciados neste estudo, foram deiscência, exsudato (seroso ou purulento) e hiperemia (20\%), fibrina (15\%), edema (10\%), calor, dor e odor $(5 \%)$. Identificou-se $20 \%$ de infecção de sítio cirúrgico entre o $7^{\circ}$ e $14^{\circ}$ dia de pós-alta hospitalar, sendo esta taxa considerada limítrofe entre os parâmetros preconizados pelo Center of Deasese Control. ${ }^{12}$ 
A identificação dos sinais e sintomas de infecção de sítio cirúrgico através de visitas domiciliares permite ao enfermeiro a execução de ações rápidas e eficazes para a resolução dos problemas identificados. Ressalta-se a necessidade de implementar um método de vigilância no pós-alta hospitalar que possibilite uma real notificação das infecções de sítio cirúrgico, já que estas podem ser subnotificadas ocasionando uma alta taxa de morbi-mortalidade.

Apesar de algumas limitações deste estudo, como o fato de ter sido realizado em apenas um hospital e sua amostra ser considerada pequena, seus resultados ressaltam a importância da vigilância das infecções de sítio cirúrgico no pós-alta hospitalar de cirurgia cardíaca reconstrutora, pois um número expressivo destas infecções manifestase fora do ambiente hospitalar.

A vigilância das ISCs, por meio de visitas domiciliares de enfermagem, integrada ao trabalho dos demais profissionais, possibilita uma prática pautada na interação e troca de experiência entre os integrantes da equipe de saúde, com vistas a oferecer uma assistência holística ao indivíduo e sua família.

\section{REFERÊNCIAS}

1. Titoto L, Sansão MS, Marino LHC, Lamari NM. Reabilitação de pacientes submetidos á cirurgia de revascularização do miocárdio: atualização da literatura nacional. Arq Ciênc Saúde. 2005 Out-Dez; 12(4):216-9.

2. Galdeano LE, Ross LA, Santos CB, Dantas RS. Diagnóstico de enfermagem no perioperatório de cirurgia cardíaca. Rev Esc Enferm USP. 2006 Mar; 40(1):26-33.

3. Pereira MS, Souza ACS, Tipple AFV, Prado MA. A infecção hospitalar e suas implicações para o cuidar da enfermagem. Texto Contexto Enferm. 2005 AbrJun; 14(2):250-7.

4. Abboud CS. Infecções em pós-operatório de cirurgia cardíaca. Rev Soc Cardiol do Estado de São Paulo. 2001 Set-Out; 11(5):915-21.

5. Secretaria de Estado da Saúde. Projeto Estadual de Prevenção de Infecção Cirúrgica - Provitae. Infecção em sítio cirúrgico [página na internet]. [acesso 2008 Fev 22] Disponível em: http://www.cve.saude. sp.gov.br/htm/ih/provitae_infeccao.htm

6. Sociedade Brasileira de Infectologia. Projeto Diretrizes. Associação Médica Brasileira e Conselho Federal de Medicina. Prevenção da infecção hospitalar [página na internet]. 2001 [acesso 2008 Mar 15] Disponível em http://www.projetodiretrizes. org.br/projeto_diretrizes/065.pdf

7. Pereira APS, Tessarini MM, Pinto MH, Oliveira VDC. Alta hospitalar: visão de um grupo de enfermeiras. Rev Enferm UERJ. 2007 Jan-Mar; 15(1):40-5.

8. Dantas RAS, Aguillar OM. Problemas na recuperação de pacientes submetidos á cirurgia de revascularização do miocárdio: o acompanhamento pelo enfermeiro durante o primeiro mês após a alta hospitalar. Rev Latino-am Enfermagem. 2001 NovDez; 9(6):31-6.

9. Oliveira AC, Ciosak SI. Infecção de sítio cirúrgico em hospital universitário: vigilância pós-alta e fatores de risco. Rev Esc Enferm USP. 2007 Jun; 41(2):258-63.

10. Carvalho ARS, Matsuda LM, Stuchi AG, Coimbra JAH. Investigando as orientações oferecidas ao paciente em pós-operatório de revascularização miocárdica. Rev Eletrônica Enferm. 2008 [acesso 2008 Out 16]; 10(2):504-12. Disponível em www.fen. ufg.br/revista/v10/n2/v10n1a21.htm

11. Mayhall CG, Mayhall CGMD. Hospital epidemiology and infection control. surgical site infections. $3^{\mathrm{a}}$ ed. United State: Lippincott Williams \& Wilkins; 2004.

12. Center of Diseases Control (CDC). Orientação para a prevenção de infecção do sitio cirúrgico [página na internet]. 1999 [acesso 2008 Fev 25]. Disponível em http://www.cdc.gov/ncidod/dhqp/pdf/ guidelines/SSI.pdf

13. Pavanello RSC, Akemi SC, Paulin LD, Lacerda RA, Uchikawa GK. A problemática do monitoramento das infecções de sítio cirúrgico e a necessidade de padronização de critérios para seu diagnóstico e notificação. Rev Eletrônica Semestral Enferm. 2005 Nov [acesso 2008 Fev 22]; (7):1-6. Disponível em: http:// revistas.um.es/eglobal/article/ view/466/492

14. Roese A, Lopes MJM. A visita domiciliar como instrumento de coleta de dados de pesquisa e vigilância em saúde: relato de experiência. Rev Gaúcha Enferm. 2004 Abr; 25(1):98-111.

15. Poveda VB. Análise dos fatores predisponentes à infecção do sítio cirúrgico em gastrectomia. [dissertação]. Ribeirão Preto (SP): Universidade de São Paulo, Escola de Enfermermagem de Ribeirão Preto; 2004.

16. Matsuda K. Hotta T, Takifuji K, Yokoyama $\mathrm{S}$, Higashiguchi $\mathrm{T}$, Tominaga $\mathrm{T}$, et al. Longterm comorbidity of diabetes mellitus is a risk factor for perineal wound complications after an abdominoperineal resection. Langenbecks Arch Surg. 2009 Jan; 394(1):65-70. 